\title{
FERRAMENTAS DE GESTÃO DE QUALIDADE COMO ESTRATÉGIAS PARA REDUÇÃO DO CANCELAMENTO E ATRASOS DE CIRURGIAS
}

Quality management tools as strategies for reducing surgery cancellations and delays

\author{
Herramientas de gestión de calidad como estrategias \\ para reducción de la cancelación y retrasos de cirugía
}

\author{
Renata Souza Souto Tamiasso ${ }^{1 *}$, Danielle Cunha Santos', Vanessa Dutra Ormundo Fernandes², \\ Cybele Aparecida Ferreira loshida ${ }^{3}$, Vanessa Brito Poveda ${ }^{4}$, Ruth Natalia Teresa Turrini ${ }^{5}$
}

RESUMO: Objetivo: Relatar a experiência de redução de cancelamentos de cirurgias que utilizou ferramentas da gestão de qualidade para solucionar a problemática de falta de materiais para as cirurgias. Método: Para o mapeamento da problemática, a equipe listou uma série de fatores relacionados que foram categorizados e apresentados na forma de diagrama de Ishikawa; posteriormente, utilizou-se a matriz de priorização de gravidade, urgência e tendência (GUT). Resultados: Por meio da otimização do "bate-mapa", da participação ativa dos seus integrantes e da adoção de ferramentas de gestão (diagrama de Ishikawa e matriz GUT), ações foram tomadas, levando à solução de fragilidades relacionadas à dinâmica cirúrgica. Conclusão: O uso de ferramentas gerenciais para detalhamento do problema e melhor utilização do "bate-mapa" cirúrgico possibilitou solucionar problemas relacionados à suspensão, ao atraso e/ou à transferência de procedimentos, mitigando problemas futuros.

Palavras-chave: Gestão da qualidade. Resolução de problemas. Enfermagem. Esterilização.

ABSTRACT: Objective: Case study on the reduction of surgery cancellations leveraging quality management tools to solve the problem of lack of surgical materials. Method: For mapping the problem, the team listed related factors that were categorized and presented using an Ishikawa diagram and, after that, the GUT Prioritization Matrix. Results: Through the optimization of the "Surgical Data Map", the active participation of its members and the adoption of management tools (Ishikawa diagram and GUT), actions were taken and led to the solution of weaknesses related to the surgical dynamics. Conclusion: The use of management tools to detail the problem and to make better use of the surgical mapping made it possible to solve several issues related to suspension, delay and transfer of procedures, thus mitigating future problems. Keywords: Quality management. Problem solving. Nursing. Sterilization.

RESUMEN: Objetivo: Informe de caso para la reducción de cancelaciones de cirugías que utilizó herramientas de gestión de calidad para solucionar la problemática de falta de materiales para las cirugías. Método: Para el mapeo de la problemática, el equipo enumeró una serie de factores relacionados que fueron categorizados y presentados en la forma de Diagrama de Ishikawa, posteriormente se utilizó la Matriz de Priorización GUT. Resultados: Por medio de la optimización del "bate-mapa", de la participación activa de sus integrantes y de la adopción de herramientas de gestión (Diagrama de Ishikawa y GUT), se tomaron acciones que llevaron a la solución de fragilidades relacionadas con la dinámica quirúrgica. Conclusión: El uso de herramientas gerenciales para detallar el problema y la mejor utilización del "bate-mapa” quirúrgico posibilitó solucionar diversos problemas relacionados con la suspensión, el retraso o la transferencia de procedimientos, mitigando los problemas futuros.

Palabras clave: Gestión de la calidad. Solución de problemas. Enfermería. Esterilización.

'Enfermeira do Centro de Material e Esterilização do Hospital Santa Catarina; especialista em Centro Cirúrgico, Recuperação Anestésica e Centro de Material e Esterilização; integrante do Grupo de Pesquisa Tecnologias e Intervenções na Saúde do Adulto com Enfoque no Paciente Cirúrgico na Escola de Enfermagem da Universidade de São Paulo (EEUSP) - São Paulo (SP), Brasil.

¿Enfermeira supervisora do Centro Cirúrgico, Endoscopia e da Central de Material e Esterilização do Instituto de Responsabilidade Social Sírio Libanês (IRSSL) - Hospital Geral do Grajaú; especialista em Doação e Transplante de Órgãos e Implantes de Tecidos; integrante do Grupo de Pesquisa Intervenções na Saúde do Adulto com Enfoque no Paciente Cirúrgico na EEUSP - São Paulo (SP), Brasil.

${ }^{3}$ Enfermeira coordenadora do Centro de Material e Esterilização do Hospital Santa Catarina; especialista em Centro Cirúrgico, Recuperação Anestésica e Centro de Material e Esterilização pela Faculdade Metropolitanas Unidas (FMU) - São Paulo (SP), Brasil.

«Enfermeira; doutora em Enfermagem Docente da EEUSP; líder do Grupo de Pesquisa Tecnologias e Intervenções na Saúde do Adulto com Enfoque no Paciente Cirúrgico na EEUSP - São Paulo (SP), Brasil. ${ }^{5}$ Enfermeira; doutora em Enfermagem; professora livre-docente da EEUSP; vice-líder do Grupo de Pesquisa Tecnologias e Intervenções na Saúde do Adulto com Enfoque no Paciente Cirúrgico na EEUSP São Paulo (SP), Brasil.

*Autora correspondente: sstrenata@gmail.com

Recebido: 24/08/2017 - Aprovado: 28/01/2018

DOI: $10.5327 / Z 1414-4425201800020007$ 


\section{INTRODUÇÃO}

O Centro de Material e Esterilização (CME) é uma unidade funcional destinada ao processamento de Produtos Para Saúde (PPS) nos serviços de saúde. As unidades de CME são classificadas como Classe I ou II, segundo a Resolução da Diretoria Colegiada (RDC) da Agência Nacional de Vigilância Sanitária (ANVISA), RDC n ${ }^{\circ}$ 15, de 15 de março de $2012^{1}$. O CME Classe I é aquele que realiza o processamento de PPS não críticos, semicríticos e críticos, de conformação não complexa, passíveis de processamento. O CME Classe II é o que executa o processamento de PPS não críticos, semicríticos e críticos, de conformação complexa e não complexa, passíveis de processamento.

O responsável técnico (RT) do CME é um profissional de nível superior legalmente habilitado, que assume perante a vigilância sanitária a responsabilidade técnica pelo serviço de saúde ou pela empresa processadora, conforme legislação vigente. ${ }^{1}$ O enfermeiro, integrante da equipe de saúde, reúne as condições imprescindíveis para assumir a responsabilidade pelo CME. É o profissional que recebe na sua formação acadêmica, tanto nos cursos de graduação como de pós-graduação, conteúdos específicos sobre as boas práticas para o processamento de PPS $^{2}$.

A unidade de CME se articula com a maioria dos setores do hospital, pois fornece PPS para setores que compreendem não só o Centro Cirúrgico (CC), mas também unidades de internação, ambulatório, emergência, terapia intensiva, entre outras ${ }^{3}$.

O foco do CME é o processamento de PPS, de modo a garantir a segurança do paciente e evitar a ocorrência de eventos adversos relacionados à utilização desses materiais ${ }^{4}$. O processamento de PPS envolve etapas sequenciais e unidirecionais, e qualquer falha não detectada em uma delas pode comprometer o resultado esperado, ou seja, um artigo seguro para o paciente, de modo a salvaguardá-lo de uma infecção relacionada à assistência à saúde (IRAS).

O ambiente hospitalar está em constante evolução, em busca de um atendimento cada vez mais seguro e de excelência. Dessa forma, novas tecnologias são incorporadas na prática diária e novas metas são estabelecidas nos diversos planejamentos estratégicos.

Com o avanço tecnológico, o desenvolvimento de novas técnicas cirúrgicas e procedimentos minimamente invasivos, os artigos e equipamentos utilizados em intervenções agressivas tornaram-se cada vez mais complexos, com a necessidade de aprimoramento dos processos de limpeza, preparo, esterilização e armazenamento dos materiais.

Assim, o principal parceiro interno do CME é o CC, por ser o responsável pelo fornecimento de materiais para atender os mais diversos procedimentos anestésico-cirúrgicos. Por esse motivo, as ações desenvolvidas no CME repercutem diretamente na dinâmica do CC. A falha em algum dos processos, além de comprometer a segurança do paciente, pode acarretar o atraso de cirurgias e até mesmo o seu cancelamento.

O cancelamento de cirurgias decorre, entre outros, da falta de planejamento. É um evento importante, que merece a devida atenção de toda a equipe envolvida na realização do procedimento anestésico-cirúrgico, principalmente quando os motivos são potencialmente evitáveis 5 .

Situações como essas envolvem ações de gestão de qualidade, a fim de manter a observância e o cumprimento de boas práticas de atenção à saúde, à eficácia e à eficiência da assistência hospitalar. As ferramentas utilizadas na gestão de qualidade para análise de eventos e tomadas de decisão permitem definir, mensurar, analisar e propor soluções para problemas que interferem direta ou indiretamente na assistência ao paciente.

A motivação em escrever esse relato se deve a uma experiência de sucesso para solucionar a falta de materiais em sala operatória (SO) e outros problemas correlatos, diminuindo o cancelamento de cirurgias por motivos não referentes ao paciente, mas intimamente ligados ao CME.

\section{OBJEtIVO}

Relatar um caso ocorrido em um CME que utilizou ferramentas da gestão de qualidade para solucionar a problemática de falta de materiais para as cirurgias e como estratégia para solução do caso foi proposta uma reformulação da reunião interna diária denominada "bate-mapa".

\section{MÉTODO}

\section{Tipo de estudo}

Trata-se de um relato de experiência que descreve a atuação de enfermeiros do CME para superar o desafio do cancelamento de cirurgias, devido à falta de determinados materiais para o ato operatório.

\section{Contexto do campo em estudo}

O cenário utilizado está inserido em uma instituição de saúde de grande porte, de atenção terciária, privada, fundada em 1906, vinculada a uma organização de saúde, situada em São Paulo. 
O trabalho realizado nesse hospital é reconhecido pela Joint Comission Internacional (JCI) e QMentum, que orientam e monitoram padrões de alta performance em qualidade. Nacionalmente é acreditado pela Organização Nacional de Acreditação (ONA), com nível de excelência, certificação máxima concedida por essa organização. Essas entidades utilizam um sistema de avaliação que, após a análise dos processos de trabalho, reconhece formalmente e certifica a qualidade dos serviços prestados. Para garantir os níveis de qualidade, anualmente as avaliações são revalidadas por equipe externa especializada.

O hospital em contexto é de alta complexidade, possui atualmente 294 leitos operacionais e 73 leitos de terapia intensiva. Abrange várias especialidades clínicas e cirúrgicas, incluindo: craniomaxilofacial, bucomaxilofacial, cardiovascular, cabeça e pescoço, aparelho digestório, geral, pediátrica, plástica, torácica, vascular, coloproctologia, neurocirurgia geral e especializada, ortopedia, oncologia, urologia, entre outras. O corpo clínico cirúrgico é aberto, porém, existe um processo para manter o relacionamento com esses profissionais, mediante um cadastramento prévio.

O CME é centralizado tipo Classe II, com área física de $295 \mathrm{~m}^{2}$, equipado com tecnologia moderna para processamento de PPS e rastreabilidade dos processos de limpeza, preparo, desinfecção e esterilização. Essa sistemática permite atender, em média, 1.200 cirurgias por mês.

\section{Elaboração do diagrama de Ishikawa}

Em 2014, o CME em questão apresentava algumas dificuldades na sua dinâmica de trabalho diária, sendo a maior delas a falta de produtos para atender a demanda das cirurgias eletivas, de urgência e de emergência, o que acarretava atrasos ou cancelamentos de procedimentos. Para o mapeamento da problemática, a equipe listou uma série de fatores relacionados, que foram categorizados e apresentados na forma de diagrama de Ishikawa, também conhecido como diagrama de causa e efeito (Figura 1).

O diagrama de Ishikawa foi utilizado para apresentar a relação existente entre o fenômeno, a causa e o efeito, que, por razões técnicas, possam afetar o resultado considerado. $\mathrm{O}$ interesse final do processo constituiu um problema a ser solucionado e então utilizado para sumarizar e apresentar as possíveis causas, atuando como um guia para identificação e determinação das medidas corretivas adotadas ${ }^{6}$.

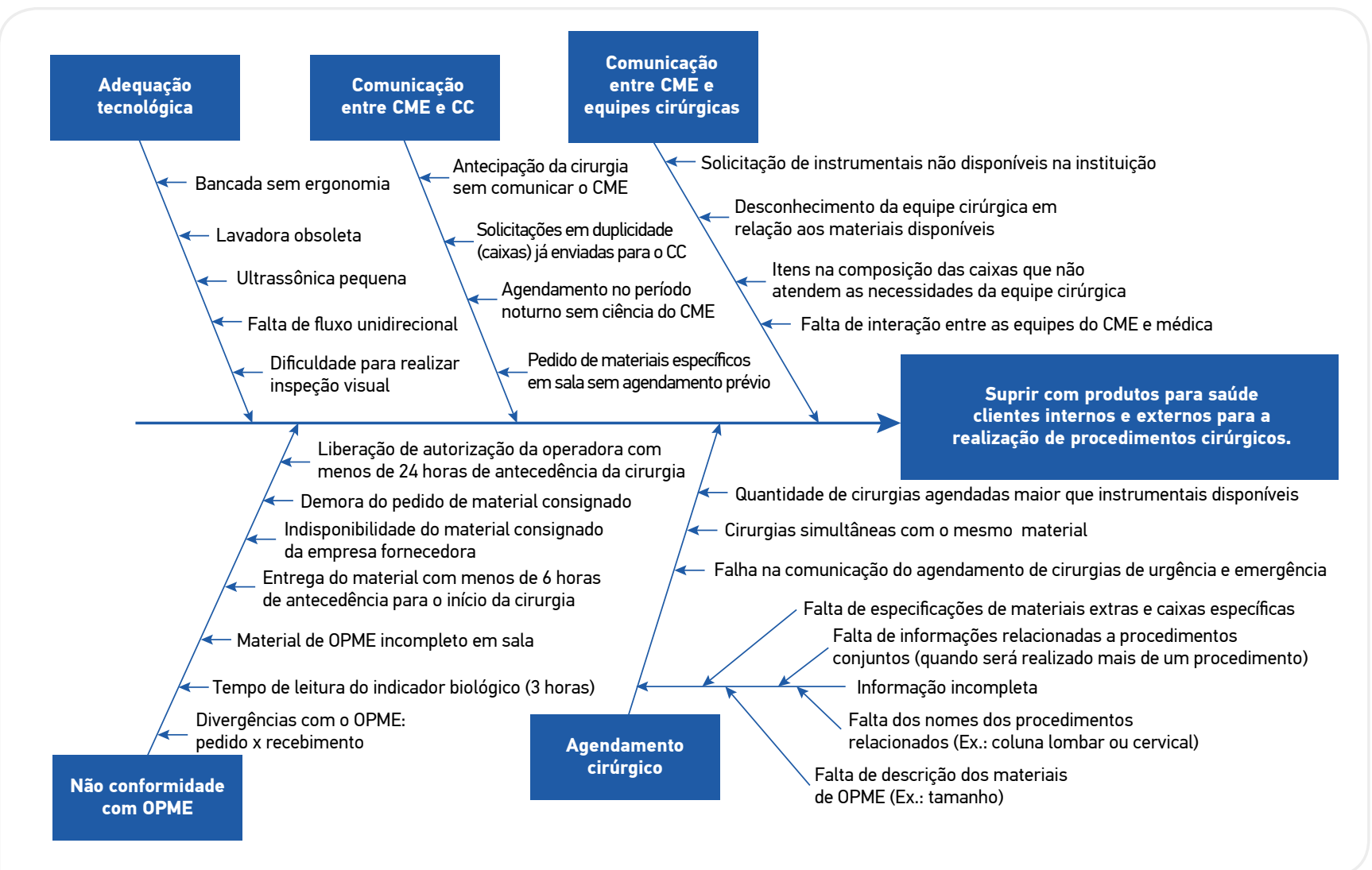

Figura 1. Diagrama de causa e efeito: mapeamento das fragilidades. 


\section{Definição de prioridades de ação}

Após a listagem dos diferentes problemas e suas inter-relações pelo diagrama de causa e efeito, identificaram-se oportunidades de melhoria. Para a resolução de problemas optou-se por utilizar a ferramenta matriz de priorização (gravidade, urgência e tendências -GUT), de modo a priorizar as ações a serem tomadas (Quadro 1).

O passo a passo para a construção da matriz incluiu:

- passo 1: formulação da lista dos problemas;

- passo 2: classificação dos problemas para cada uma das três variáveis (GUT). Cada problema foi analisado com uma nota de 1 a 5 para cada característica, sendo 1 para casos sem gravidade, sem urgência e tendência a piorar, e 5 para os extremamente graves, urgentes e que se não forem resolvidos pioram imediatamente;

- passo 3: identificação do ranking dos principais problemas (multiplicando as três notas);

- passo 4: análise para localização dos pontos fracos (ranking dos principais problemas);

- passo 5: elaboração dos planos de ação para solucionar ou diminuir os problemas ${ }^{6}$

\section{Proposta para solução}

Após a análise dos problemas, detectaram-se outras causas correlatas, que necessitavam de soluções em curto e médio

Quadro 1. Matriz de priorização.

Descrição do Problema

Material de OPME ${ }^{\star *}$ incompleto em sala

Falta de descrição dos materiais de OPME (Ex.: tamanho)

Pedido de materiais específicos em sala sem agendamento prévio

Cirurgias simultâneas com o mesmo material

Lavadora obsoleta

Divergências com o OPME: pedido $x$ recebimento

Falta fluxo unidirecional

Quantidade de cirurgias agendadas maior que instrumentais disponíveis

Falta de informações relacionadas a procedimentos conjuntos

Falha na comunicação do agendamento de cirurgias de urgência e emergência

Indisponibilidade do material consignado da empresa fornecedora

Solicitação de instrumentais não disponíveis na instituição

Entrega do material com menos de 6 horas de antecedência para o início da cirurgia

Antecipação da cirurgia sem comunicar o CME

Agendamento no período noturno sem ciência do CME

Demora do pedido de material consignado

Falta dos nomes dos procedimentos relacionados (Ex.: coluna lombar ou cervical)

Dificuldade para realizar inspeção visual

Itens na composição de caixas que não atendem as necessidades da equipe cirúrgica

Falta de especificações de materiais extras e caixas específicas

Ultrassônica pequena

Falta de interação entre as equipes do CME e médica

Tempo de leitura do indicador biológico (3 horas)

Liberação da autorização da operadora com menos de 24 horas de antecedência da cirurgia

Desconhecimento da equipe cirúrgica em relação aos materiais disponíveis

Bancada sem ergonomia

Solicitações em duplicidades (caixas já enviadas para o CC)

\begin{tabular}{|c|c|c|c|c|}
\hline G & $\mathbf{U}$ & $\mathbf{T}$ & SCORE & * \\
\hline 5 & 5 & 5 & 125 & 10 \\
\hline 5 & 5 & 5 & 125 & $2^{\circ}$ \\
\hline 4 & 5 & 5 & 100 & $3^{\circ}$ \\
\hline 5 & 4 & 4 & 80 & $4^{\circ}$ \\
\hline 5 & 4 & 4 & 80 & $5^{\circ}$ \\
\hline 4 & 4 & 4 & 64 & $6^{\circ}$ \\
\hline 4 & 4 & 4 & 64 & $7^{\circ}$ \\
\hline 4 & 3 & 3 & 36 & $8^{\circ}$ \\
\hline 4 & 3 & 3 & 36 & 90 \\
\hline 3 & 4 & 3 & 36 & $10^{\circ}$ \\
\hline 4 & 3 & 3 & 36 & $11^{\circ}$ \\
\hline 3 & 4 & 3 & 36 & $12^{\circ}$ \\
\hline 3 & 4 & 3 & 36 & $13^{\circ}$ \\
\hline 3 & 3 & 3 & 27 & $14^{\circ}$ \\
\hline 3 & 3 & 3 & 27 & $15^{\circ}$ \\
\hline 3 & 3 & 3 & 27 & $16^{\circ}$ \\
\hline 3 & 3 & 3 & 27 & $17^{\circ}$ \\
\hline 4 & 3 & 2 & 24 & $18^{\circ}$ \\
\hline 3 & 2 & 3 & 18 & $19^{\circ}$ \\
\hline 3 & 2 & 3 & 18 & $20^{\circ}$ \\
\hline 2 & 3 & 3 & 18 & $21^{\circ}$ \\
\hline 2 & 3 & 2 & 12 & $22^{\circ}$ \\
\hline 2 & 2 & 2 & 8 & $23^{\circ}$ \\
\hline 2 & 2 & 2 & 8 & $24^{\circ}$ \\
\hline 2 & 2 & 2 & 8 & $25^{\circ}$ \\
\hline 2 & 2 & 2 & 8 & $26^{\circ}$ \\
\hline 2 & 2 & 2 & 8 & $27^{\circ}$ \\
\hline
\end{tabular}

*Priorização; OPME: Órtese, Próteses e Materiais Especiais. 
prazos. No entanto, esses problemas envolviam outros setores, além do CME, e a interação entre eles possibilitaria solucionar a maioria dos itens levantados. Identificaram-se três frentes de ações para solucionar a problemática:

1. "bate-mapa";

2. investimento financeiro nas seguintes frentes: adequação de infraestrutura, aquisição de novas tecnologias, instrumentais cirúrgicos e aumento no quadro de recursos humanos;

3. comunicação interprofissional.

\section{RESULTADOS}

\section{"Bate-Mapa"}

Os enfermeiros do CME começaram a utilizar de forma sistemática uma reunião denominada "bate-mapa", que acontecia todos os dias para verificar as autorizações dos procedimentos cirúrgicos. Faziam parte dessa reunião os enfermeiros do CME, do CC, do Agendamento Cirúrgico e um Técnico de Enfermagem que realiza a cobrança dos materiais consignados. A reunião diária possibilitou a identificação de outros fatores e a adoção de soluções efetivas.

Com essa nova reformulação da reunião diária, "bate-mapa”, somaram-se a esse grupo espontaneamente os farmacêuticos do CC, o enfermeiro da hemodinâmica e, em algumas reuniões específicas, a liderança do setor de materiais consignados. Atualmente, os colaboradores novos dos setores citados, aqueles em fase de treinamento e enfermeiros trainees também participam da reunião do "bate-mapa".

Por meio da otimização do "bate-mapa", da participação ativa dos seus integrantes e da adoção de ferramentas de gestão, várias ações foram tomadas, o que levou à solução de fragilidades, exemplificadas no Quadro 2.

Para algumas adequações, foi necessário envolver o setor financeiro da instituição, como descrito no Quadro 3.

Também identificaram-se importantes componentes de comunicação interprofissional, como as listadas a seguir, no Quadro 4.

As ações implantadas resultaram em soluções que contribuíram para melhoria do indicador de cancelamento de cirurgias. Os dados foram selecionados segundo o critério da falta de material fornecido pelo CME. Analisando esse indicador, verificou-se que, nos últimos 5 anos, a taxa de

Quadro 2. Ações tomadas a partir do "bate-mapa".

Remanejamento do horário de cirurgias simultâneas com o mesmo material.

Atendimento das solicitações do material em sala é respeitado de acordo com o agendamento prévio e disponibilidade do mesmo.

Solicitações de OPME são realizadas logo após a autorização da solicitação.

Consulta a outras empresas fornecedoras quando a indisponibilidade do material consignado da empresa de primeira escolha em situações de urgência e emergência, conforme critérios estabelecidos previamente.

Realização da conferência do material de acordo com a solicitação médica e autorização do convênio no ato da entrega do consignado.

Solicitação do material pelo setor de consignados por contato telefônico e e-mail à empresa fornecedora. Em caso de atraso, o enfermeiro do CME realiza uma nova ligação telefônica para nova previsão de entrega do material.

OPME: Órtese, Próteses e Materiais Especiais; CME: Centro de Material e Esterilização.

Quadro 3. Investimento financeiro nas seguintes áreas: adequação de infraestrutura, aquisição de novas tecnologias, instrumentais cirúrgicos e aumento no quadro de recursos humanos.

Aquisição de novos materiais mediante o perfil de agendamento cirúrgico.

Aquisição de indicador biológico com leitura de 1 hora.

Aquisição de seis bancadas e cadeiras de regulação automática conforme estatura do colaborador.

Aquisição de 3 termodesinfectoras.

Aquisição de 1 ultrassônica com maior capacidade.

Adequação na estrutura física para manter o fluxo unidirecional.

Aquisição de microscópio para inspeção e troca de sistema de iluminação.

Contratação de um coordenador para o CME e dois enfermeiros exclusivos no plantão noturno.

CME: Centro de Material e Esterilização. 
Quadro 4. Comunicação interprofissional.

Comunicação ao CME a ocorrência de procedimentos de urgência e emergência via telefone e e-mail pelo setor de agendamento cirúrgico. Revisão das informações dos agendamentos quando necessário, pelo enfermeiro responsável por este setor. Antecipação do horário da cirurgia, somente após a verificação da disponibilidade de instrumentais e consignados pelo enfermeiro do CME. Registro das caixas enviadas ao CC em documento denominado "Aviso Cirúrgico", onde conta toda informação de materiais e equipamentos para a cirurgia de um paciente. A informação da caixa também é inserida em um sistema informatizado de rastreabilidade.

Convite às equipes cirúrgicas para conhecer os materiais pertinentes a sua especialidade disponíveis no arsenal do CME, para melhorar o gerenciamento.

Reformulação da composição das caixas existentes conforme sugestão dos cirurgiões e instrumentadores. Aquisição de novas caixas definidas em parceria com enfermeiros do CME e equipes cirúrgicas.

Presença dos enfermeiros do CME em SO durante o período Perioperatório quando necessário, em contato direto com a equipe multidisciplinar, para esclarecer dúvidas ou apresentar propostas para solução de problemas imediatos relacionados aos instrumentais.

Dúvidas ao receber materiais consignados, a equipe cirúrgica é contatada para maiores esclarecimentos.

CME: Centro de Material e Esterilização; CC: Centro Cirúrgico; SO: sala de operação

cancelamentos atingiu seu ápice em $2012(3,8 \%)$, pior resultado em decorrência desse problema, e foi decaindo ao longo dos anos, apresentando o seu melhor desempenho em 2016, atingindo menos de $0,5 \%$ de cancelamentos dos procedimentos anestésico-cirúrgicos agendados.

\section{DISCUSSÃO}

No intuito de avaliar a qualidade dos serviços de saúde, é fundamental a escolha de um método para que sejam estabelecidas diretrizes ou padrões que auxiliem no alcance dos mais elevados níveis de excelência. Na prática hospitalar, as instituições optam pelos processos de acreditação, que são voluntários e desenvolvidos de forma periódica, segundo a Portaria do Ministério da Saúde (MS) n ${ }^{\circ} 538$, de 17 de abril de 2001, que reconhece a ONA como instituição competente e autorizada a operacionalizar o desenvolvimento do Processo de Acreditação Hospitalar ${ }^{7}$.

Esse processo permite gerenciar os serviços de saúde com qualidade, segundo as recomendações do Manual de Acreditação Hospitalar ${ }^{7,8}$. Como se trata de um programa de avaliação, espera-se a adoção de indicadores, pois são medidas objetivas que permitem, por meio dos seus resultados, evidenciar a realidade. Dessa forma também é possível identificar novas práticas. No período de avaliação constatou-se a importância de não apenas monitorar o indicador, mas também intervir quando necessário. Para isso, é essencial a adoção de ferramentas que auxiliem nas tomadas de decisões, como as utilizadas neste relato de experiência, que incluiu o diagrama de Ishikawa e a matriz de priorização GUT.

A gestão do indicador "cancelamento de cirurgias" permitiu às equipes do CME e multidisciplinar, buscar soluções para diversas questões. Um estudo demonstrou que o cancelamento de cirurgias repercute em toda a cadeia de serviços no processo anestésico-cirúrgico. A falta de materiais é relatada como uma das maiores causas que corroboram esse cenário 9 .

\section{CONCLUSÃO}

Este relato permitiu evidenciar uma melhor interação entre os vários setores de um grande hospital, por meio da reunião diária denominada "bate-mapa". Essa prática possibilitou solucionar problemas, como a falta de materiais para atender as cirurgias, evitando a suspensão, o atraso ou a transferência de procedimentos, mitigando diversos problemas futuros.

Verificou-se que a adoção de ferramentas de gestão de qualidade pôde auxiliar na tomada de decisões, com aumento da qualidade gerencial, assistencial e a humanização das relações com os clientes. Todos os profissionais envolvidos se conscientizaram, sem exceção, de que são corresponsáveis tanto pelos resultados positivos de um procedimento bemsucedido como pelos desperdícios, prejuízos e retrabalho. Além disso, o enfermeiro, perante a equipe multidisciplinar, é o profissional com maior engajamento e conhecimento para coordenar um grupo de trabalho para a melhoria assistencial e segurança do paciente. 


\section{REFERÊNCIAS}

1. Brasil. Agência Nacional de Vigilância Sanitária. Resolução RDC $n^{\circ} 15$, de 15 de março de 2012. Dispõe sobre requisitos de boas práticas para o processamento de produtos para saúde e dá outras providências. Brasília: ANVISA; 2012.

2. Associação Brasileira de Enfermeiros de Centro Cirúrgico, Recuperação Anestésica e Centro de Material e Esterilização. Diretrizes de práticas em enfermagem cirúrgica e processamento de produtos para a saúde. $7^{a}$ ed. Barueri: Manole/São Paulo: SOBECC; 2017.

3. Silva A. Organização do centro de materiais e esterilização. In: Graziano KU, Silva A, Psaltikidis EM, orgs. Enfermagem em centro de material e esterilização. Barueri: Manole; 2011. p. 1-21.

4. Padoveze MC, Graziano KU. Limpeza, desinfecção e esterilização de artigos em serviços de saúde. São Paulo: APECIH; 2010. p. 1-33.
5. Ávila MAG, Gonçalves IR, MartinsI, Moyses AM. Cancelamento de cirurgias: uma revisão integrativa da literatura. Rev SOBECC. 2012;17(2):39-47.

6. Couto RC, Pedrosa TMG. Hospital: acreditação e gestão em saúde. $2^{a}$ ed. Rio de Janeiro: Guanabara Koogan; 2007.

7. Brasil. Ministério da Saúde. Portaria GM no 538, de 17 de abril de 2001. Brasília: Ministério da Saúde; 2001.

8. Brasil. Organização Nacional de Acreditação. Manual Brasileiro de Acreditação: serviços para a saúde selo de qualificação ONA. Brasília: Organização Nacional de Acreditação; 2016.

9. Nascimento LA, Tillvitz LR, Fonseca LF. Suspensão cirúrgica: o ângulo estatístico de um problema de repercussões humanas. Rev Enferm UFPE. 2013;7(Esp.):6592-600. DOI: 10.5205/ reuol.5058-41233-3-SM.071 lesp201305 